\title{
ESTIMATED CRITICALITY LOWER-LIMIT MULTIPLICATION FACTOR OF LOW-ENRICHED URANIUM DIOXIDE-CONCRETE SYSTEM USING THE BOOTSTRAP METHOD
}

\author{
Takuto HAYASHI $^{1}$, Fuga NISHIOKA ${ }^{1}$, Tomohiro ENDO ${ }^{1}$, and Akio YAMAMOTO ${ }^{1}$ \\ ${ }^{1}$ Nagoya University \\ Furo-cho, Chikusa-ku, Nagoya, Aichi, Japan, 464-8603 \\ t-hayashi@fermi.energy.nagoya-u.ac.jp,f-nishioka@fermi.energy.nagoya-u.ac.jp, \\ t-endo@energy.nagoya-u.ac.jp, a-yamamoto@energy.nagoya-u.ac.jp
}

\begin{abstract}
The present paper aims to evaluate the estimated criticality lower-limit multiplication factor of fuel debris in a form of uranium dioxide-concrete mixture for a study of criticality control on the fuel debris generated through the molten core concrete interaction in a severe accident of a light water reactor. The estimated criticality lower-limit multiplication factor is evaluated using the bootstrap method where the assumption of the normal distribution is not necessary. In addition, it is calculated taking into account correlation coefficients that represent the degrees of neutronic similarity between the target system and benchmark critical experiment systems, experimental uncertainties of benchmark data, and statistical uncertainties of calculated effective multiplication factor by a continuous energy Monte Carlo code. This paper shows that the estimated criticality lower-limit multiplication factor using the bootstrap method can be comparable with a baseline upper-subcritical-limit which is evaluated by Whisper-1.1 without margins of subcriticality for uncertainties from nuclear covariance data and undetected errors in software.
\end{abstract}

KEYWORDS: estimated criticality lower-limit multiplication factor, fuel debris, MCCI, bootstrap method, baseline upper-subcritical-limit

\section{INTRODUCTION}

The estimated criticality lower-limit multiplication factor (ECLLMF) is the upper limit of the neutron multiplication factor where the target system may be judged subcritical in consideration of calculation errors [1]. It is an important parameter to determine the upper subcritical limit for nuclear criticality safety. Many benchmark calculations using critical experiments which are physically similar to the target system are carried out; and calculated effective multiplication factors $\left(k_{\text {eff }}\right)$ obtained by benchmark critical experiments are statistically processed to evaluate the ECLLMF.

A statistical process of $k_{\text {eff }}$ to evaluate the ECLLMF has been developed using the bootstrap method where the assumption of the normal distribution is not necessary [2]. In addition, a calculation method of the ECLLMF was proposed taking into account (1) correlation coefficients that represent the degrees of neutronic similarity between the target system and benchmark critical experiment systems, (2) experimental uncertainties of benchmark data, and (3) statistical uncertainties of calculated $k_{\text {eff }}$ by a continuous energy Monte Carlo code [3], [4]. 
It is highly probable that a mixture of damaged fuel and concrete has been generated through the molten core-concrete interaction in a severe accident of a light water reactor. Concrete is mainly composed of a mixture of silicon dioxide $\left(\mathrm{SiO}_{2}\right)$; and it contains light elements that are important from the viewpoint of criticality, e.g., hydrogen and carbon. Izawa et al. reported that concrete provided efficient neutron moderation and pointed out the necessity of further investigation on the criticality of uranium dioxide $\left(\mathrm{UO}_{2}\right)$-concrete system for the actual handling of fuel debris [5].

The purpose of this study is evaluation of the ECLLMF of fuel debris in a form of $\mathrm{UO}_{2}$-concrete mixture for a study of criticality control on the fuel debris. As a step to achieve the purpose, we examine the number of critical experiments, which are physically similar to a $\mathrm{UO}_{2}$-concrete system, archived in the International Criticality Safety Benchmark Evaluation Project (ICSBEP) Handbook [6]. In addition, we assess the normality of the calculated $k_{\text {eff }}$ obtained by the benchmark critical experiments. Finally, we evaluate the ECLLMF using the bootstrap method since calculated $k_{\text {eff }}$ does not obey the normal distribution. The ECLLMF using the bootstrap method, the ECLLMF using the normality assumption, and a baseline upper-subcritical-limit evaluated by Whisper-1.1 [7] are compared.

\section{THEORY}

\subsection{Estimated Criticality Lower-Limit Multiplication Factor with the Normality Assumption}

The ECLLMF can be calculated using the properties of the normal distribution and the non-central $t$ distribution based on statistical theory when a probability distribution of calculated $k_{\text {eff }}$ can be regarded to obey the normal distribution [1]. Then the ECLLMF with the normality assumption $k_{\text {sub,t }}$ is expressed by:

$$
k_{\mathrm{sub}, \mathrm{t}}=\bar{k}-\alpha_{\mathrm{t}} s
$$

where

$$
\begin{gathered}
\bar{k}=\frac{1}{n} \sum_{i=1}^{n} k_{\mathrm{eff}, i}, \\
s^{2}=\frac{1}{n-1} \sum_{i=1}^{n}\left(k_{\mathrm{eff}, i}-\bar{k}\right)^{2},(n \geq 2) .
\end{gathered}
$$

Note that $\bar{k}$ is the sample mean of $k_{\mathrm{eff}, 1}, k_{\mathrm{eff}, 2}, \ldots, k_{\mathrm{eff}, n}$ obtained by the benchmark calculations for $n$ critical experiments; $\alpha_{\mathrm{t}}$ is a margin value, and $s$ is the sample standard deviation of $k_{\mathrm{eff}, 1}, k_{\mathrm{eff}, 2}, \ldots, k_{\mathrm{eff}, n}$.

The margin value of $\alpha_{\mathrm{t}}$ can be calculated by setting $p$ (excess probability of critical condition) and $\gamma$ (success probability of setting ECLLMF). The value of $p$ and $\gamma$ are set individually by an analyst, e.g., $p=0.025$ and $\gamma=0.975$. A detailed calculation method of $\alpha_{\mathrm{t}}$ is described in Ref. [2].

\subsection{Estimated Criticality Lower-Limit Multiplication Factor Using the Bootstrap Method}

In the previous study [2], the ECLLMF using the bootstrap method without the assumption of the normal distribution has been developed. In addition, a calculation method of ECLLMF was developed within the consideration of (1) correlation coefficients $\left(c_{k}\right)$ that represent the degrees of neutronic similarity between 
a target system and benchmark critical experiment systems, (2) experimental uncertainties of benchmark data $\left(\sigma_{\text {bench }}\right)$, and (3) statistical uncertainties of calculated $k_{\text {eff }}\left(\sigma_{\text {calc }}\right)$ by a continuous energy Monte Carlo code [3], [4]. Calculation procedures of the latter ECLLMF $k_{\text {sub,boot }}$ are explained as follows:

1. The experimental and statistical uncertainties of $k_{\mathrm{eff}, i}$ are synthesized by:

$$
\sigma_{i}=\sqrt{\sigma_{\text {bench }, i}^{2}+\sigma_{\text {calc }, i}^{2}}
$$

If the experimental and statistical uncertainties of $k_{\text {eff }}$ are not considered, $\sigma_{i}$ is set to be sufficiently small $\left(\sigma_{i}=10^{-8}\right)$.

2. A multi-modal distribution $p(x)$ is defined by a mixture of weighted $n$ normal distributions:

$$
p(x)=\sum_{i=1}^{n} \frac{\omega_{i}}{\sqrt{2 \pi \sigma_{i}^{2}}} \exp \left(-\frac{\left(x-k_{\mathrm{eff}, i}\right)^{2}}{2 \sigma_{i}^{2}}\right)
$$

where the parameter $\omega_{i}$ is the normalized weighting factor corresponding to the representative factor $c_{k, i}$ of the benchmark critical experiment. If the representative factors are not considered, $\omega_{i}=1 / n$, i.e., each of critical experiments has the same weight. The representative factor $c_{k, i}$ is calculated by the nuclear data-induced correlation factor, of which the detailed calculation method is described in Ref. [7]. The weighting factor $w_{i}$ and the normalized weighting factor $\omega_{i}$ are given by, respectively:

$$
\begin{gathered}
w_{i}=\frac{c_{k, i}-c_{k, \mathrm{acc}}}{c_{k, \max }-c_{k, \mathrm{acc}}}, \\
\omega_{i}=\frac{w_{i}}{\sum_{i=1}^{n} w_{i}^{\prime}}
\end{gathered}
$$

where the subscript $i$ indicates the $i$-th critical experiment; $c_{k, \max }$ is the maximum value of $c_{k, i}$; and $c_{k \text {,acc }}$ is the acceptable minimum value of $c_{k}$. The value of $c_{k \text {,acc }}$ is determined so that a summation of the weighting factors $w_{i}$ is greater than or equal to $w_{\text {rep }}$, which is an adequate total weight of the sample size. In Whisper-1.1, the value of $w_{\text {rep }}$ is evaluated as follows [7]:

$$
w_{\text {req }}=w_{\min }+w_{\text {penalty }}\left(1-c_{k, \max }\right)
$$

where $w_{\min }$ is the minimum number of neutronically identical benchmark critical experiments, and $w_{\text {penalty }}$ is a penalty factor for not having a benchmark critical experiment that is neutronically identical to the target system. The default values are $w_{\min }=25$ and $w_{\text {penalty }}=100$ in Whisper-1.1.

3. Calculate the lower $100 p$ percentile of the multi-modal distribution expressed by Eq. (5). The lower $100 p$ percentile is defined by the value of a random variable where the value of the cumulative distribution function is $p$. Hereafter, the percentile is called $k_{\mathrm{eff}, p}$. For example, $k_{\mathrm{eff}, p}$ can be evaluated using the bisection method.

4. Generate a new set of resamples $k_{1}^{* b}, k_{2}^{* b}, \ldots, k_{n}^{* b}$ by the parametric bootstrap sampling based on the multi-modal distribution, where the superscript ' $* b$ ' means the $b$-th bootstrap sample. To consider 
the representative factors in this bootstrap sampling, the normalized weighting factor $\omega_{i}$ is used as the probability to select for the corresponding normal distribution $\mathcal{N}\left(k_{\mathrm{eff}, i}, \sigma_{i}^{2}\right)$.

5. Calculate the bootstrap sample mean $\hat{k}^{* b}$ and the bootstrap sample standard deviation $\hat{s}^{* b}$ for the $b$-th set of resamples, respectively.

6. Obtain $\hat{\mathbf{k}}^{*}=\left(\hat{k}^{* 1}, \hat{k}^{* 2}, \ldots, \hat{k}^{* B}\right)$ and $\hat{\mathbf{s}}^{*}=\left(\hat{s}^{* 1}, \hat{s}^{* 2}, \ldots, \hat{s}^{* B}\right)$ by repeating the procedures $4-5$ in $B$ times.

7. $\mathbf{k}_{\text {sub,boot }}=\left(k_{\text {sub,boot }}^{1}, k_{\text {sub,boot }}^{2}, \ldots, k_{\text {sub,boot }}^{B}\right)$ is obtained by:

$$
\mathbf{k}_{\text {sub,boot }}=\hat{\mathbf{k}}^{*}-\alpha_{\text {boot }} \hat{\mathbf{s}}^{*}
$$

where the margin value $\alpha_{\text {boot }}$ is determined so that the probability of $\mathbf{k}_{\text {sub,boot }}$ exceeding $k_{\text {eff, } p}$ is $1-\gamma$ using the bisection method.

8. The ECLLMF $k_{\text {sub,boot }}$ is finally obtained by:

$$
k_{\text {sub,boot }}=\bar{k}-\alpha_{\text {boot }} S \text {, }
$$

where the parameters $\bar{k}$ and $s$ are the mean and the standard deviation of the multi-modal distribution expressed by Eq. (5), respectively.

\section{ESTIMATED CRITICALITY LOWER-LIMIT MULTIPLICATION FACTOR OF LOW- ENRICHED URANIUM DIOXIDE-CONCRETE SYSTEM}

\subsection{Calculation Conditions}

The ECLLMF was calculated for an infinite lattice of a sphere cell. As shown in Figure 1, the sphere cell is composed of an inner $\mathrm{UO}_{2}$ sphere with a radius of $1.00 \mathrm{~cm}$ and surrounded by a $1.07 \mathrm{~cm}$ thickness of concrete moderator with the white boundary condition. The fuel has a ${ }^{235} \mathrm{U}$ enrichment of $5 \mathrm{wt} . \%$ and contains no burnable poison. In addition, the moderation ratio is almost optimum. Compositions of fuel and concrete are shown in Table I. The temperature of the fuel and concrete are set to be $293 \mathrm{~K}$.

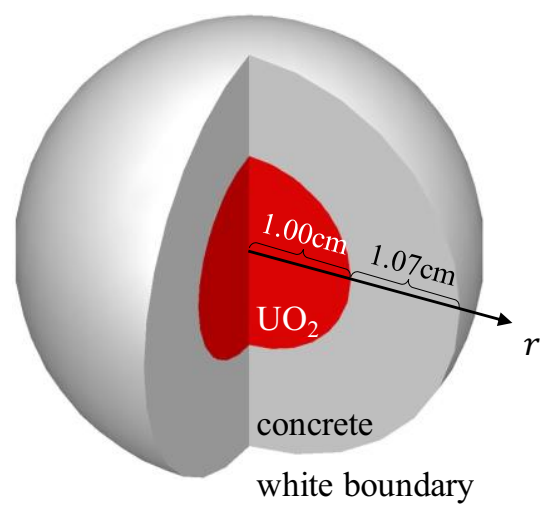

Figure 1. Cell Geometry and Boundary Condition. 
Table I. Compositions of Fuel and Concrete.

\begin{tabular}{|c|l|l|}
\hline & Nuclide & $\begin{array}{c}\text { Atomic number density } \\
\left(\times 10^{24} \text { atoms } / \mathrm{cm}^{3}\right)\end{array}$ \\
\hline \multirow{4}{*}{$\begin{array}{c}\text { Fuel } \\
\text { (5 wt.\% })\end{array}$} & ${ }^{235} \mathrm{U}$ & $1.1757 \times 10^{-3}$ \\
\cline { 2 - 3 } & ${ }^{238} \mathrm{U}$ & $2.2057 \times 10^{-2}$ \\
\cline { 2 - 3 } & $\mathrm{O}$ & $4.6465 \times 10^{-2}$ \\
\hline & $\mathrm{H}$ & $1.3742 \times 10^{-2}$ \\
\cline { 2 - 3 } & $\mathrm{O}$ & $4.5921 \times 10^{-2}$ \\
\cline { 2 - 3 } & $\mathrm{C}$ & $1.1532 \times 10^{-4}$ \\
\cline { 2 - 3 } Concrete & $\mathrm{Na}$ & $9.6397 \times 10^{-4}$ \\
\cline { 2 - 3 }$\left(2.3 \mathrm{~g} / \mathrm{cm}^{3}\right)$ & $\mathrm{Mg}$ & $1.2389 \times 10^{-4}$ \\
\cline { 2 - 3 } & $\mathrm{Al}$ & $1.7409 \times 10^{-3}$ \\
\cline { 2 - 3 } & $\mathrm{Si}$ & $1.6617 \times 10^{-2}$ \\
\cline { 2 - 3 } & $\mathrm{K}$ & $4.6054 \times 10^{-4}$ \\
\cline { 2 - 3 } & $\mathrm{Ca}$ & $1.5026 \times 10^{-3}$ \\
\cline { 2 - 3 } & $\mathrm{Fe}$ & $3.4507 \times 10^{-4}$ \\
\hline
\end{tabular}

Note: "Isotopic abundances are natural.

Whisper-1.1 [7] uses benchmark critical experiments from the ICSBEP Handbook [6], nuclear data sensitivities from a continuous energy Monte Carlo transport software MCNP6.2, and nuclear covariance data ENDF/B-VII.1 to select critical experiments which are physically similar to the target system. The critical experiments whose representative factor $c_{k, i}$ is larger than the acceptable value of $c_{k \text {,acc }}$ are selected by Whisper-1.1. The value of $c_{k, \text { acc }}$ and the maximum value of $c_{k, i}\left(c_{k, \max }\right)$ for the target system are 0.5975 and 0.8764 , respectively. The normalized weighting factors for each critical experiment are summarized in Figure 2. The total number of critical experiments $(n)$ is 118.

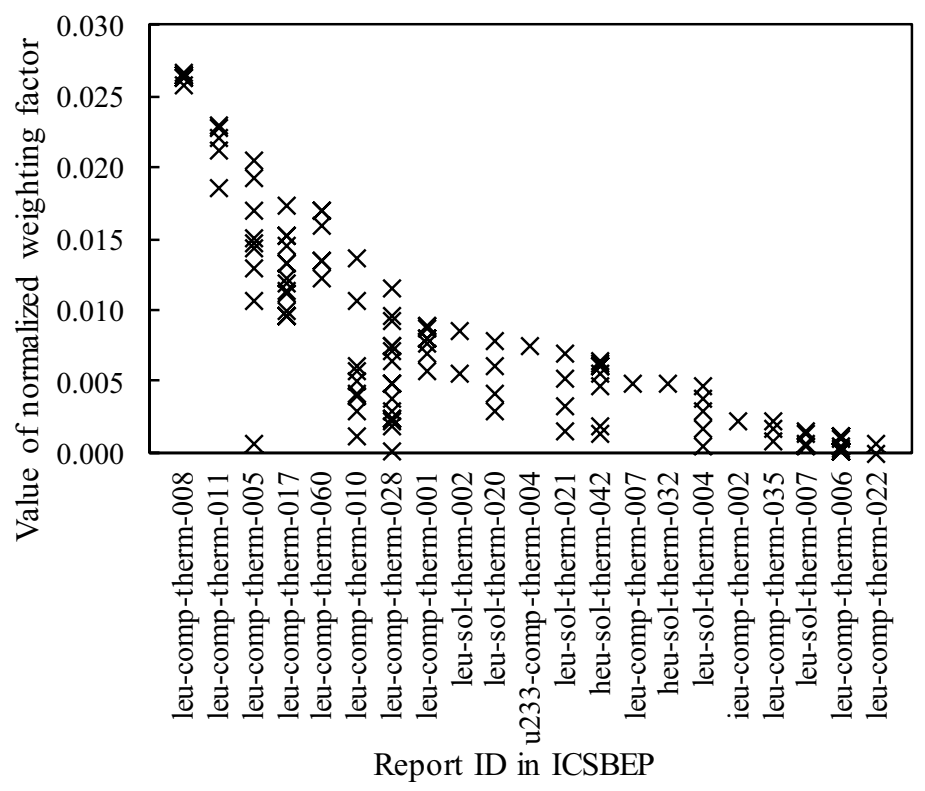

Figure 2. Normalized Weighting Factors for each Critical Experiment. 


\subsection{Estimation Results of Estimated Criticality Lower-Limit Multiplication Factor}

The values of $p$ (excess probability of critical condition) and $\gamma$ (success probability of setting ECLLMF) were set to be 0.01 and 0.99 , respectively. The total number of bootstrap procedures $(B)$ was $10^{5}$. The effective multiplication factors recorded in Whisper-1.1 were corrected by subtracting the bias from the calculated $k_{\text {eff }}$ value. Here, the bias is defined by subtracting unity from the experimental $k_{\text {eff }}$ value. Figure $3 \mathrm{a}$ shows a calculation result of ECLLMF without consideration of neutronic similarity and uncertainties of $k_{\text {eff }}$, i.e., $\omega_{i}=1 / n$ and $\sigma_{i}=10^{-8}$. Figure $3 \mathrm{~b}$ shows a calculation result of ECLLMF taking the neutronic similarity and uncertainties of $k_{\text {eff }}$ into account, as explained in Section 2.2. Note that $\mathbf{k}_{\text {sub,boot }}$ in Figure 3 means a histogram of bootstrap distribution itself, not ECLLMF. Values of $\bar{k}, s$, $k_{\mathrm{eff}, p}, \alpha$ and ECLLMF are summarized in Table II.

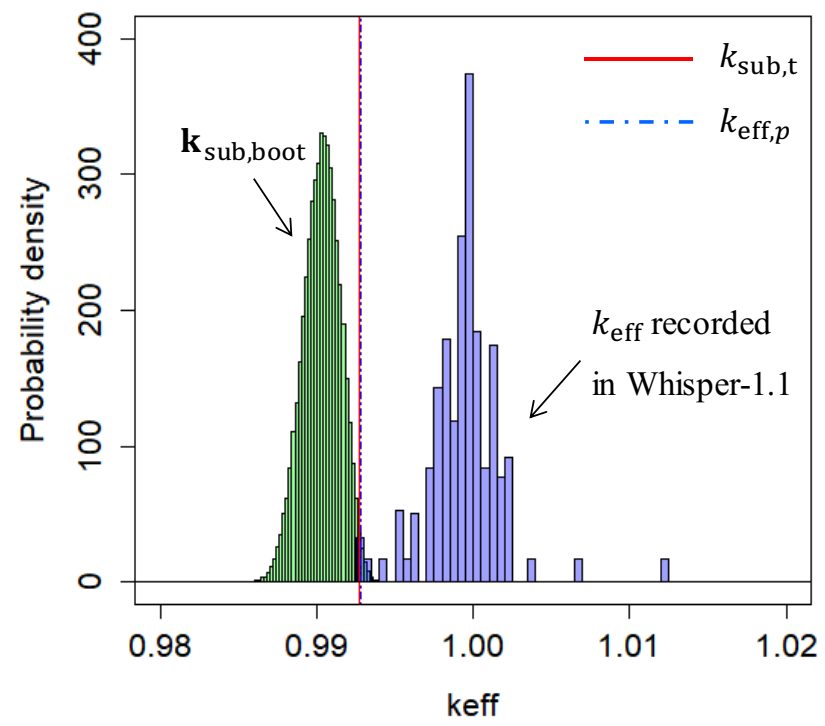

(a) Without neutronic similarity and uncertainties of effective multiplication factor

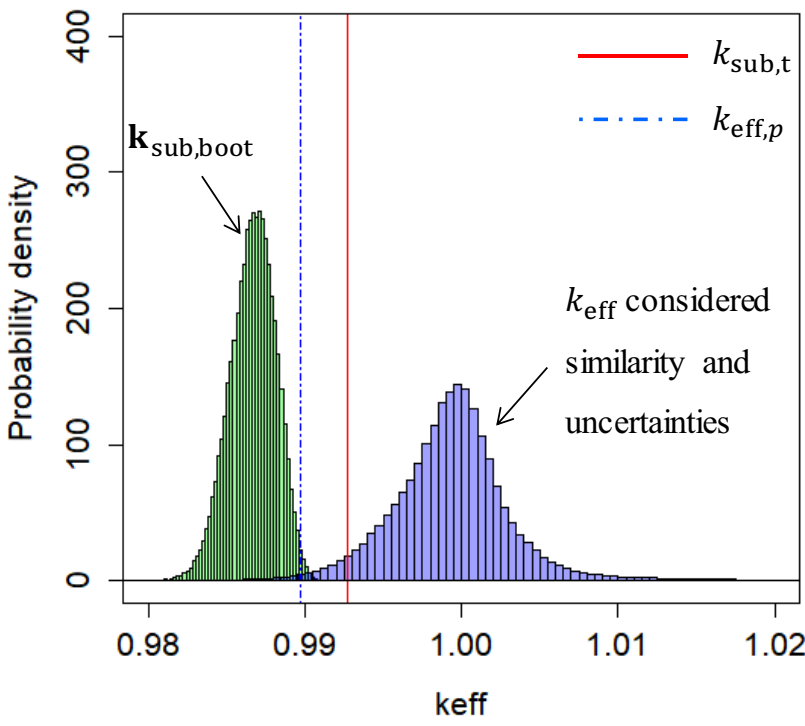

(b) With neutronic similarity and uncertainties of effective multiplication factor

Figure 3. Probability Density Distribution of Calculated Effective Multiplication Factor and Bootstrap Distribution (a) without Consideration of Neutronic Similarity and Uncertainties of Effective Multiplication Factor and (b) with Consideration of Neutronic Similarity and Uncertainties of Effective Multiplication Factor.

Table II. Summary of Estimated Criticality Lower-Limit Multiplication Factor.

\begin{tabular}{|l|c|c|c|}
\hline & $\begin{array}{c}\text { Normality assumption } \\
\text { corresponding to } k_{\text {sub,t }}\end{array}$ & $\begin{array}{c}\text { Bootstrap method } \\
\text { corresponding to Fig. 3a }\end{array}$ & $\begin{array}{c}\text { Bootstrap method } \\
\text { corresponding to Fig. 3b }\end{array}$ \\
\hline $\bar{k}$ & 0.99939 & 0.99939 & 0.99937 \\
\hline$s$ & 0.00239 & 0.00238 & 0.00399 \\
\hline$k_{\text {eff }, p}$ & 0.99384 & 0.99283 & 0.98969 \\
\hline$\alpha$ & 2.8015 & 3.8611 & 3.2081 \\
\hline ECLLMF & 0.99270 & 0.99022 & 0.98657 \\
\hline
\end{tabular}


The estimated values of $\bar{k}$ and $s$ are the same, respectively, in calculation methods based on the normality assumption and the bootstrap method without neutronic similarity and uncertainties of $k_{\text {eff }}$; this is caused that the random variable $x$ of the multi-modal distribution expressed by Eq. (5) with $\omega_{i}=1 / n$ and $\sigma_{i}=$ $10^{-8}$ is consequently the same as calculated $k_{\mathrm{eff}, i}$.

An extension of Shapiro and Wilk's $W$ test [8], which is a normality test, was carried out for a probability density distribution of $k_{\text {eff }}$ and the obtained p-value was $8.87 \times 10^{-8}$. Since the p-value was smaller than 0.05 , it was confirmed that the probability density distribution of $k_{\text {eff }}$ did not obey the normal distribution. Therefore the ECLLMF using the bootstrap method is more reasonable than the ECLLMF with the normality assumption in this study.

In the case of Whisper-1.1, a baseline upper-subcritical-limit (USL) is calculated by:

$$
\mathrm{USL}=1-\beta-\sigma_{\beta}-2.6 \mathrm{MOS}_{\text {data }}-\mathrm{MOS}_{\text {software }}-\mathrm{MOS}_{\text {application }}
$$

where $\beta$ is the discrepancy of a weighted average of calculated $k_{\text {eff }}$ from its expected value, called the bias; $\sigma_{\beta}$ is the quadrature sum of the weighted experimental and statistical uncertainties, called the bias uncertainty; $\mathrm{MOS}_{\text {data }}$ is a margin of subcriticality for nuclear data uncertainties; $\mathrm{MOS}_{\text {software }}$ is a margin of subcriticality for undetected errors in software; and $\mathrm{MOS}_{\text {application }}$ is a margin of subcriticality for the application.

The values of parameters for USL of a $\mathrm{UO}_{2}$-concrete system as shown in Figure 1 are summarized in Table III. The USL is calculated by Whisper-1.1. The value $1-\beta-\sigma_{\beta}$ in Table III is an uppersubcritical-limit with consideration of neutronic similarity and uncertainties of $k_{\text {eff }}$, and note that this value does not include margins of nuclear data uncertainties and undetected errors in software. Thus, the value $1-\beta-\sigma_{\beta}$ was utilized for comparison with ECLLMF as presented in Table II.

Table III. Summary of Upper-Subcritical-Limit Obtained by Whisper-1.1.

\begin{tabular}{|c|l|}
\hline$\beta$ & 0.00944 \\
\hline$\sigma_{\beta}$ & 0.00821 \\
\hline MOS $_{\text {data }}$ & 0.00183 \\
\hline MOS $_{\text {software }}$ & 0.00500 \\
\hline MOS $_{\text {application }}$ & 0.00000 \\
\hline $1-\beta-\sigma_{\beta}$ & 0.98235 \\
\hline USL & 0.97260 \\
\hline
\end{tabular}

The estimated value of ECLLMF using the bootstrap method was slightly larger than $1-\beta-\sigma_{\beta}$ by $0.00422[\Delta k]$ when neutronic similarity and uncertainties of $k_{\text {eff }}$ were considered. This difference was caused by the difference of the evaluation method between ECLLMF and USL, i.e., USL was evaluated based on the extreme value theory.

\section{CONCLUSIONS}

The present paper aimed to evaluate the estimated criticality lower-limit multiplication factor (ECLLMF) of fuel debris in a form of uranium dioxide $\left(\mathrm{UO}_{2}\right)$-concrete mixture. The ECLLMF was evaluated taking 
into account correlation coefficients that represent the degrees of neutronic similarity between the target system and benchmark critical experiment systems, experimental uncertainties of benchmark data, and statistical uncertainties of calculated effective multiplication factor by a continuous energy Monte Carlo code using the bootstrap method where the assumption of the normal distribution was not necessary.

In the case of this study, the ECLLMF for a low-enriched $\mathrm{UO}_{2}$-concrete system was approximately 0.987 . This estimated value was comparable with a baseline upper-subcritical-limit which was evaluated by Whisper-1.1 without margins of subcriticality for uncertainties from nuclear covariance data and undetected errors in software.

Our future research is the development of a calculation method for the ECLLMF to consider the nucleardata-induced uncertainties.

Furthermore, fuel debris in an actual situation is submerged in water, and the composition of concrete could be influenced by the process of the molten core-concrete interaction. Therefore, further studies are also needed considering those situations for the handling of fuel debris.

\section{REFERENCES}

1. "Nuclear Criticality Safety Handbook, 2; English Translation," JAERI-Review-2001-028 (2001).

2. T. Hayashi, T. Endo, and A. Yamamoto, "Calculation Method of Estimated Criticality Lower-Limit Multiplication Factor Using the Bootstrap Method," Proc. Mathematics and Computational Methods Applied to Nuclear Science and Engineering (M\&C 2019), Portland, USA, Aug. 25-29, 2019, American Nuclear Society (2019).

3. T. Hayashi, F. Nishioka, T. Endo, and A. Yamamoto, "Estimated Criticality Lower-Limit Multiplication Factor Considering Neutronic Similarity and Uncertainties of Effective Multiplication Factor Using the Bootstrap Method (1) Theory," Proc. Reactor Physics Asia 2019 (RPHA19), Osaka, Japan, Dec. 2-3, 2019 (2019).

4. F. Nishioka, T. Hayashi, T. Endo, and A. Yamamoto, "Estimated Criticality Lower-Limit Multiplication Factor Considering Neutronic Similarity and Uncertainties of Effective Multiplication Factor Using the Bootstrap Method (2) Application," Proc. Reactor Physics Asia 2019 (RPHA19), Osaka, Japan, Dec. 2-3, 2019 (2019).

5. K. Izawa, Y. Uchida, K. Ohkubo, M. Totsuka, H. Sono, and K. Tonoike, "Infinite Multiplication Factor of Low-Enriched $\mathrm{UO}_{2}$-Concrete System," J. Nucl. Sci. Technol., 49(11), pp. 1043-1047 (2012).

6. "International Handbook of Evaluated Criticality Safety Benchmark Experiments," https://www.oecdnea.org/science/wpncs/icsbep/handbook.html (2018).

7. B. C. Kiedrowski, F. B. Brown, J. L. Conlin, J. A. Favorite, A. C. Kahler, A. R. Kersting, D. K. Parsons, and J. L. Walker, "Whisper: Sensitivity/Uncertainty-Based Computational Methods and Software for Determining Baseline Upper Subcritical Limits," Nucl. Sci. Eng., 181(1), pp. 17-47 (2015).

8. J. P. Royston, "An Extension of Shapiro and Wilk's $W$ Test for Normality to Large Samples," Appl. Statist., 31(2), pp. 115-124 (1982). 